\title{
ASPECTOS METROLÓGICOS DA TERMOGRAFIA NA INDÚSTRIA DE PETRÓLEO
}

Bruna Beatriz de Freitas ${ }^{1}$, Ricardo Savio T. Moretz Sohn, Mario Anselmo Pereira Neto

${ }^{1}$ Instituto Nacional de Metrologia, Qualidade e Tecnologia - Inmetro

bbfreitas-petrobras@inmetro.gov.br

Submetido 10/10/2016 - Aceito 22/02/2017

DOI: 10.15628/holos.2016.xxxx

\section{RESUMO}

Câmeras térmicas, também denominadas termovisores, vêm se tornando uma alternativa para medição de temperatura em situações em que, tradicionalmente, eram utilizados termômetros de contato como termopares, termorresistores e até mesmo termômetros de não contato, também chamados de termômetros de infravermelho ou pirômetros. Entretanto, apesar das vantagens das câmeras térmicas, como, por exemplo, a velocidade de resposta bem superior à dos termômetros de contato, ou da possibilidade de se obter a distribuição de temperaturas de uma superfície de interesse, a obtenção de medidas confiáveis de temperatura com esses termovisores é um tanto mais complexa, exigindo do operador, conhecimentos tais como a emissividade efetiva da superfície alvo, o que não é necessário na termometria de contato. Além disso, plantas na área de petróleo e gás, como é o caso de refinarias ou plataformas de petróleo são, em geral, instalações complexas e de grande extensão, o que pode exigir a utilização de diversas câmeras térmicas. A confiabilidade nas medições com esses equipamentos, e a comparação entre as medições com termovisores diferentes, requer que essas medições sejam realizadas seguindo recomendações metrológicas e que as medições incluam o valor de suas respectivas incertezas. $O$ objetivo deste artigo é abordar os aspectos metrológicos, incluindo o cálculo da incerteza de medição, nas avaliações termográficas em situações típicas da indústria de petróleo e proporcionar um maior domínio aos usuários desses termovisores para a obtenção de medições mais confiáveis.

PALAVRAS-CHAVE: termografia, câmeras térmicas, incerteza, indústrias de petróleo, termometria.

\section{THERMOGRAPHY METROLOGICAL ASPECTS IN OIL INDUSTRY}

\begin{abstract}
Thermal imaging cameras, also called thermal imagers, are becoming an alternative for temperature measurement in situations where traditionally were used other contact (e.g. thermocouples) and noncontact thermometers (e.g. infrared thermometers). Some of their advantages are the faster response in comparison with contact thermometers and the possibility of acquiring the temperature distribution of a surface. However, obtaining reliable temperature measurements with these instruments is somewhat more complex. It is of fundamental importance, for example, knowing the effective emissivity of the target surface, which is not necessary for contact
\end{abstract}

thermometry. In addition, plants in oil and gas industry, like refineries or oil rigs, are in general complex and large installations, which may require the use of several thermal cameras. To rely on and compare their measurements, it is necessary that they are carried out following metrological recommendations and that their respective uncertainties are correctly evaluated. The objective of this paper is to address the metrological aspects, including uncertainty evaluation, of thermal imaging cameras in typical situations of the oil industry, so that their users can obtain more reliable measurements.

KEYWORDS: thermography, thermal cameras, uncertainty, oil industry, thermometry. 


\section{INTRODUÇÃO}

A utilização de técnicas de medição de temperatura sem contato em indústrias de petróleo tem aumentado consideravelmente em função de diversos fatores como redução no preço dos equipamentos, aumento de qualidade e resolução destes e facilidade na obtenção da temperatura aparente (temperatura indicada pelo equipamento), diminuindo tempo gasto para a realização das medições. Uma dessas técnicas é chamada de termografia, a qual capta radiação térmica emitida pelos objetos e a converte em uma imagem, permitindo a visualização da distribuição de temperatura da região ou objeto de interesse, por meio de um instrumento denominado de câmera térmica ou termovisor (Gaussorgues, 1994).

Câmeras térmicas possuem inúmeras aplicações nas plantas de petróleo e gás como verificar e monitorar combustão em refinarias de petróleo detectando se houve a queima de poluentes como o metano ou se o mesmo foi lançado na atmosfera (Flir, S.d), detectar superaquecimento em sistemas (Fluke, 2000), verificar o nível ou quantidade de produto disponível em tanques (Clausing, 2008), detectar falhas no revestimento refratário interno de um regenerador de uma planta de craqueamento catalítico fluido, identificar falhas no isolamento térmico de queimadores de caldeiras flamotubulares (Neto, 2004), detectar escapamento de gases em tubulações, etc. A termografia também é utilizada em manutenções preditivas evitando paradas desnecessárias dos processos de produção, maximizando a eficiência desses processos, e também pode ser utilizada em qualquer situação em que a temperatura seja um indicativo importante de uma possível falha (Xavier, 2005).

Apesar de seu papel importante em diversas situações como as acima mencionadas, percebe-se que a bibliografia sobre o assunto é rica em exemplos de aplicação da termografia, mas carece de maiores informações a respeito das grandezas que influenciam o resultado de medição bem como fontes que contribuem para a incerteza de medição. No Brasil, o serviço de calibração de termovisores ainda está em fase de implantação pelo Inmetro e mesmo em âmbito internacional ainda não há consenso quanto aos métodos de calibração de câmeras térmicas (Mcevoy, Simpson, \& Machin, 2012). Apesar de haver fabricantes de termovisores de reconhecida qualidade no mercado nacional e internacional, o estado incipiente da rede metrológica nacional, nessa área, leva a um questionamento acerca da confiabilidade das medições e resultados quantitativos por meios termográficos.

O conhecimento das fontes de erros e de incertezas de medição, por parte dos operadores e usuários desses termovisores, é importante por possibilitar a obtenção de medições mais confiáveis, desde que sejam tomadas medidas com intuito de minimizar as fontes de erro presentes. Esses cuidados são ainda mais necessários nos casos em que pequenas variações de temperaturas interferem significativamente no processo.

Ao longo deste artigo serão apresentadas as principais fontes de influências no resultado de medição com câmeras térmicas. Também serão considerados os impactos dos parâmetros declarados pelos fabricantes na incerteza de medição e em quais condições os mesmos são válidos. Será apresentado também um modelo matemático para o cálculo da incerteza de medição, considerando condições laboratoriais.

\section{REVISÃO BIBLIOGRÁFICA}




\subsection{Erro e incerteza de medição}

Em metrologia, uma importante fonte de referência terminológica desenvolvida com o propósito de ser uma referência comum a cientistas, engenheiros e pessoas envolvidas na realização de medições, é o Vocabulário Internacional de Metrologia (VIM). Atualmente, a versão do VIM em língua portuguesa é a 1ạ edição Luso-Brasileira (Instituto Nacional de Metrologia Qualidade e Tecnologia [Inmetro], 2012) que se baseia na 3a edição internacional do VIM (Comitê Internacional de Pesos e Medidas [BIPM], 2012). Neste artigo, quando nos referirmos ao VIM, estaremos tratando da edição em língua portuguesa. Listamos a seguir alguns importantes conceitos retirados do VIM que serão utilizados neste trabalho:

- Grandeza: Propriedade dum fenômeno dum corpo ou duma substância, que pode ser expressa quantitativamente sob a forma dum número e duma referência.

- Valor de referência duma grandeza: Valor duma grandeza utilizado como base para comparação com valores de grandezas da mesma natureza.

- Mensurando: Grandeza que se pretende medir.

- Incerteza de medição: Parâmetro não negativo que caracteriza a dispersão dos valores atribuídos a um mensurando;

- Erro de medição: Diferença entre o valor medido duma grandeza e um valor de referência.

Apesar de sua definição simples, a obtenção do erro de medição é mais complexa, pois as grandezas envolvidas nessa definição geralmente não são obtidas diretamente, e sim através de outras grandezas das quais dependem de uma forma nem sempre trivial. Além disso, essa relação de dependência nem sempre é completamente conhecida ou pode ser expressa de forma analítica.

Uma das componentes do erro de medição é o erro sistemático, que pode ser obtido a partir de medições repetidas do mensurando. Uma vez tendo sido quantificado o erro sistemático, o valor medido do mensurando deve ser corrigido de modo a compensar esse erro. Já a incerteza de medição, expressa a ideia de que o valor estimado do mensurando, mesmo após todas as correções necessárias, não é o único valor possível que pode ser razoavelmente atribuído ao mensurando. Antes, é um dos possíveis valores que o mensurando pode assumir, com uma certa probabilidade, caso fossem realizadas infinitas medições sob as mesmas condições.

A declaração da incerteza de medição possibilita a comparação dos resultados de medição de diferentes câmeras térmicas. 


\subsection{Grandezas de influência}

As grandezas de influência são aquelas que não são o mensurando, mas que podem afetar o seu resultado de medição. Vamos considerar dois tipos de grandezas de influência no caso das medições com câmeras térmicas: grandezas externas e internas.

As grandezas internas estão relacionadas com os sistemas internos da câmera, capazes de influenciar o resultado de medição, como por exemplo, emissão de radiação pelos componentes ópticos, ruídos e não linearidade do detector, variações no sinal do circuito amplificador, resolução e linearidade do conversor $A / D$, variação e exatidão limitada da curva de calibração, etc (Chrzanowski, Ficher, \& Matyszkiel, 2000).

As grandezas externas relacionam-se com a incerteza na determinação da emissividade efetiva da superfície medida, da temperatura efetiva do ambiente e da transmissividade efetiva do ambiente (Chrzanowski, Fisher, Matyskiel, \& Barela, 2001). As especificações técnicas declaradas pelos fabricantes contêm parâmetros que podem ser utilizados para estimar as grandezas de influência internas. Esses parâmetros são obtidos pelos fabricantes em condições de calibração específicas, portanto é necessário o conhecimento prévio dessas condições antes da sua utilização, pois esses parâmetros podem diferir quando as condições de medição forem diferentes das condições de calibração.

As condições de calibração atendem aos seguintes critérios:

1. A superfície medida é suficientemente larga e comporta-se, aproximadamente, como um corpo negro;

2. A distância entre a câmera térmica e a superfície medida é pequena (menos de $5 \mathrm{~m}$ ), de modo que influência da transmissividade atmosférica pode ser desprezada;

3. A temperatura ambiente está compreendida entre a faixa tipicamente encontrada em laboratórios: entre $20^{\circ} \mathrm{Ce} 30^{\circ} \mathrm{C}$;

4. A área de medição está posicionada no centro do campo de visão da câmera térmica;

5. As medições são realizadas com o ajuste de escala da câmera térmica selecionada para a escala mais curta, que compreenda a temperatura da superfície medida;

6. Havendo o ajuste correspondente ao tempo de integração, este deve ser utilizado para redução de ruído (normalmente ajustado para o tempo de integração máximo).

\section{METODOLOGIA}

Uma avaliação da câmera térmica atendendo às condições de calibração como exposto anteriormente, possibilita calcular a incerteza de medição devido, somente, às grandezas de influência que chamamos de internas, visto que as influências externas se tornam bastante reduzidas. 
A metodologia utilizada para o desenvolvimento desse trabalho consistiu na definição da equação de medição para uma câmera térmica e na identificação de um grupo de parâmetros que pudessem assumir valores com base em medições e em especificações técnicas declaradas pelos fabricantes, a partir dos quais fosse possível calcular as incertezas de medição devidas às fontes de erro internas.

Após a definição desses parâmetros, foram calculadas as incertezas de medição com base nas especificações típicas de uma câmera térmica para diversas temperaturas. Com isso, pretende-se observar o comportamento da incerteza de medição em função de valores diferentes de temperatura.

\subsection{Equação de medição}

O procedimento de medição das câmeras térmicas, que é similar ao utilizado por termômetros de radiação, é ilustrado na Figura 1.

O detector (individual) gera um sinal de saída em função da radiação recebida. Esse detector foi calibrado inicialmente na fábrica, obedecendo às condições de calibração descritas anteriormente e, portanto, o fabricante obteve a função resposta do detector que relaciona o sinal de saída com a temperatura de corpo negro, ou seja,

$$
S_{c a l}=f\left(T_{c n}\right)
$$

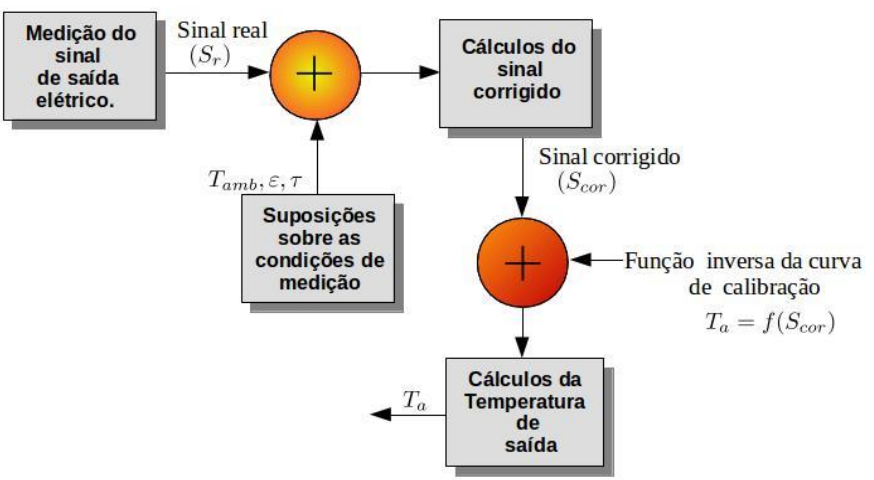

Figura 1: Procedimento de Medição de Temperatura com câmeras térmicas.

Para permitir a utilização da câmera fora das condições de calibração, o usuário deve fornecer o valor de alguns parâmetros indicativos das condições de medição. Esses parâmetros podem incluir a emissividade estimada da superfície avaliada, a temperatura ambiente onde a câmera encontra-se posicionada, a umidade relativa e a transmissividade da atmosfera entre o objeto em medição e a câmera.

Com base nos parâmetros relativos às condições de medição, correções são incorporadas ao sinal real, gerado pelo detector, de modo que, o sinal corrigido, seja obtido. Esse seria o sinal gerado caso o objeto fosse um corpo negro na mesma temperatura do objeto real.

E finalmente, a função inversa da função resposta obtida na calibração é utilizada para se obter a temperatura aparente do objeto.

Um diagrama representativo das temperaturas efetivas importantes na medição termográfica é apresentado na Figura 2, em que é indicado o fato de que nem toda a radiação 
térmica captada pelo termovisor é proveniente da radiação emitida pela superfície alvo da medição.

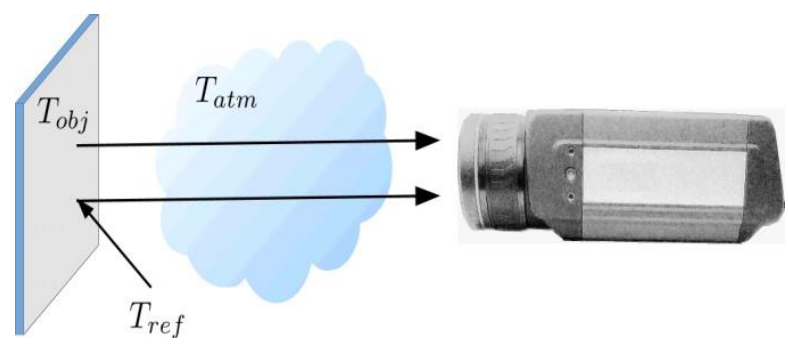

Figura 2: Diagrama esquemático representando a radiação emitida pelo objeto em medição e a radiação refletida pelo objeto em medição.

É possível modelar o processo de medição termográfica considerando o sinal real, gerado pelos detectores como sendo composto da contribuição do sinal decorrente da radiação emitida pelo objeto em medição, $S_{o b j}$, do sinal gerado pela radiação refletida no objeto, $S_{r e f}$ e do sinal gerado em função da radiação dos componentes ópticos, $S_{\text {opt }}$, de modo que se pode escrever:

$$
S_{r}=S_{o p t}+S_{o b j}+S_{r e f}
$$

A equação (2) pode ser reescrita se levarmos em conta os sinais $S_{\mathrm{en}}$ que seriam gerados num detector caso as fontes de radiação térmica fossem corpos negros ideais:

$$
S_{r}=\varepsilon \tau S_{c n}\left(T_{o b j}\right)+(1-\varepsilon) \tau S_{c n}\left(T_{r e f}\right)+S_{o p t}
$$

Como o sinal $S_{c m}\left(T_{o b j}\right)$ é o que estamos chamando de $S_{c o r}$ na Figura 1, podemos reescrever (3) isolando $S_{\text {corr }}$ :

$$
S_{c o r}=\frac{S-(1-\varepsilon) \tau S_{c n}\left(T_{r e f}\right)-S_{o p t}}{\varepsilon \tau}
$$

Finalmente, a temperatura aparente do objeto é calculada com base na função inversa da função resposta da câmera obtida em calibração.

A equação (4) nos traz algumas grandezas de influência que iremos considerar para o cálculo da incerteza de medição de modo que se pode escrever a incerteza combinada na medição da temperatura aparente, $u_{\odot}\left(T_{a}\right)$ :

$$
u_{c}\left(T_{a}\right)=\sqrt{u_{\varepsilon}^{2}+u_{T_{r e f}}^{2}+u_{\tau}^{2}+u_{i n t}^{2}}
$$


ou seja, as contribuições para a incerteza na temperatura aparente são a incerteza devido à emissividade efetiva, à temperatura refletida efetiva, à transmissividade atmosférica e a incerteza devida às fontes internas de erro.

Cada uma dessas quatro contribuições para a incerteza na grandeza de saída é o produto da incerteza padrão na grandeza de entrada pelo seu coeficiente de sensibilidade, de modo que a incerteza combinada pode ser escrita como:

$$
\left[u_{c}\left(T_{a}\right)\right]^{2}=\left[c_{\varepsilon} u(\varepsilon)\right]^{2}+\left[c_{\text {Tref }} u\left(T_{\text {ref }}\right)\right]^{2}+\left[c_{\tau} u(\tau)\right]^{2}+\left[c_{i n t} u\left(\Delta T_{\text {in }}\right)\right]^{2}
$$

Cada um dos coeficientes de sensibilidade em (6) são calculados por meio de derivadas parciais da função que permite obter a temperatura aparente $T_{a}$ em função da temperatura real do objeto $T_{o b j}$ e dos sinais $S_{\text {cor }}$ e $S_{c n}$. Para a consideração razoável de que a diferença entre $S_{c m}\left(T_{\circ b j}\right)$ e $S_{c o r}\left(T_{\circ b j}\right)$ é pequena, a relação entre $T_{a}$ e $T_{\circ b j}$ pode ser obtida assim:

$$
T_{a}=T_{o b j}+\frac{S_{c o r}\left(T_{o b j}\right)-S_{c n}\left(T_{o b j}\right)}{\left.\frac{d S_{c n}}{d T}\right|_{T=T_{o b j}}}
$$

Os coeficientes de sensibilidade para $\varepsilon, T_{\text {ref }}, \tau$ e $\Delta T_{\text {int }}$ são calculados como a seguir:

$$
\begin{gathered}
c_{\varepsilon}=\frac{\partial T_{a}}{\partial \varepsilon} \\
c_{\text {Tref }}=\frac{\partial T_{a}}{\partial T_{r e f}} \\
c_{\tau}=\frac{\partial T_{a}}{\partial \tau} \\
c_{i n t}=\frac{\partial T_{a}}{\partial \Delta T_{i n t}}
\end{gathered}
$$

que, utilizando de (8) a (11), obtém-se:

$$
c_{\varepsilon}=-\frac{I_{1}\left(T_{a}\right)-I_{1}(\operatorname{Tref})}{\varepsilon c_{2} I_{2}\left(T_{a}\right)}
$$




$$
\begin{gathered}
c_{T_{r e f}}=-\frac{(1-\varepsilon) I_{2}\left(T_{r e f}\right)}{\varepsilon I_{2}\left(T_{a}\right)} \\
c \tau=-\frac{\varepsilon I_{1}\left(T_{a}\right)-(1-\varepsilon) I_{1}\left(T_{r e f}\right)}{\varepsilon \tau c_{2} I_{2}\left(T_{a}\right)} \\
c_{\text {int }}=1
\end{gathered}
$$

As equações (12) a (14) fazem referência às funções $I_{1}(T)$ e $I_{2}(T)$ que são definidas por:

$$
\begin{gathered}
I_{1}(T)=\int_{0}^{\infty} \frac{R_{(\lambda)}}{\lambda^{5}\left[\exp \left(\frac{c_{2}}{\lambda T}\right)-1\right]} d \lambda \\
I_{2}(T)=\int_{0}^{\infty} \frac{R_{(\lambda)} \exp \left(\frac{c_{2}}{\lambda T}\right)}{\lambda^{6} T^{2}\left[\exp \left(\frac{c_{2}}{\lambda T}\right)-1\right]^{2}} d \lambda
\end{gathered}
$$

em que $R_{\lambda}$ é a responsividade espectral relativa da câmera térmica, que nesse artigo foi modelada como sendo unitária dentro dos limites da faixa espectral da câmera e nula fora desses limites.

Uma vez que (12) a (15) foram obtidos, vamos prosseguir obtendo as incertezas padrões respectivas.

Uma avaliação da incerteza de medição, com base nas fontes internas de erro, pode ser realizada se essas fontes forem representadas pelo conjunto de parâmetros, conforme seguem descritos abaixo (Chrzanowski et al., 2000):

- Erro mínimo (EM);

- Erro gerado por ruído (EGR);

- Resolução de temperatura digital (RTD);

- Estabilidade de temperatura (ET);

- Repetibilidade (RE);

- Uniformidade de medição (UM).

Na próxima seção serão discutidos esses parâmetros e apresentados os resultados envolvendo a incerteza de medição a partir deles.

\section{RESULTADOS E DISCUSSÃO}


O EM equivale ao que os fabricantes costumam designar por accuracy nos manuais das câmeras térmicas. Chamaremos aqui de erro mínimo visto que nem sempre as condições de medição correspondem às condições de calibração, de modo que a medição poderá agregar erros maiores do que os declarados como accuracy. Cabe aqui uma breve discussão sobre o conceito desse termo nos contextos da indústria e da metrologia.

Accuracy pode ser encontrado em alguns manuais em língua portuguesa traduzidos por exatidão. Entretanto, segundo o VIM (Inmetro, 2012), a exatidão não pode ser associada a números, é apenas um conceito qualitativo, de modo que não há uma concordância semântica entre a terminologia industrial e a metrológica para esse termo.

Nos catálogos dos fabricantes, accuracy é um parâmetro que provê informações acerca da incerteza de medição que pode ser obtida se atendidas às condições de calibração de fábrica. A mesma é considerada como o erro na temperatura de medição do corpo negro (fonte de radiação térmica utilizada em calibração), nas condições de calibração e quando a temperatura do corpo negro variar dentro do alcance de medição da câmera. Esse parâmetro é comumente expresso em ${ }^{\circ} \mathrm{C}$ ou \% da indicação.

Para uma dada câmera térmica, o seguinte valor de accuracy foi fornecido: $\pm 1{ }^{\circ} \mathrm{C}$ ou $\pm 1 \%$ (o que for maior). Nesse caso, para uma temperatura medida pelo termovisor de $300{ }^{\circ} \mathrm{C}$ obtémse um $\mathrm{EM} /{ }^{\circ} \mathrm{C}=300 \times 2 \%=6$. Note que foi usado $2 \%$ por representar a variação equivalente a $\pm 1 \%$.

O EGR, para câmeras de uma única banda espectral, é equivalente ao NETD (sigla em inglês para noise equivalent temperature difference), que é a diferença de temperatura equivalente ao ruído e também é traduzido por sensibilidade térmica ou resolução térmica. É uma figura de mérito utilizada para quantificar a razão sinal-ruído em câmeras térmicas e representa a menor diferença de temperatura que pode ser detectada pela câmera.

Esse parâmetro provê informações acerca do ruído elétrico em erros de medições e pode ser tratado como uma boa estimativa da incerteza devido a ruídos elétricos no sistema. Normalmente esse parâmetro é fornecido na unidade $\mathrm{mK}$.

O EGR (mais conhecido como NGE, que é a sigla em inglês) é em função da temperatura do objeto, mas o NETD é declarado para uma única temperatura do objeto, geralmente $30{ }^{\circ} \mathrm{C}$, logo para se obter o valor do EGR para o valor da temperatura desejada, deve-se utilizar a razão entre a integração das derivadas parciais da lei de Planck em função da temperatura do objeto e da temperatura ambiente, como demostra a Equação (18):

$$
N E T D \cdot \frac{\int_{\Delta \lambda} \frac{\partial L\left(\lambda, T_{m}\right)}{\partial T}}{\int_{\Delta \lambda} \frac{\partial L\left(\lambda, T_{o b j}\right)}{\partial T}}
$$

O RTD é a diferença entre dois níveis de temperatura que podem ser distinguidos por causa da resolução limitada do canal digital da câmera térmica, essa diferença é em função do alcance de medição da câmera $\left(\Delta T_{\text {span }}\right)$ e também do número de bits $(b)$ da mesma, logo:

$$
R T D=\frac{\Delta T_{\text {span }}}{2^{b}}
$$

A ET é definida como o alcance dos resultados de medição para diferentes temperaturas ambientes. Este parâmetro geralmente não é declarado pelos fabricantes, portanto existem duas formas de obtê-lo: a primeira é a forma experimental, que é de realização complexa e demorada, 
necessitando de câmara especial com a temperatura controlada; o segundo modo é através do cálculo da indicação de temperatura em função da temperatura ambiente, Equação 20, que foi forma utilizada neste artigo.

$$
E T=\frac{T_{o b j} \int_{\lambda 1}^{\lambda 2}\left[R(\lambda) \exp \left(c_{2} / \lambda T_{o b j}\right)\right] / \lambda^{6} T_{o b j}^{2}\left[\exp \left(c_{2} / \lambda T_{o b j}\right)-1\right]^{2} d \lambda}{\int_{\lambda 1}^{\lambda 2}[R(\lambda)] / \lambda^{5}\left[\exp \left(c_{2} / \lambda T_{o b j}\right)-1\right] d \lambda}
$$

A repetibilidade é definida pelo Inmetro (2012) como a "Precisão de medição sob um conjunto de condições de repetibilidade". A RE é obtida através do desvio padrão amostral do número de medições realizada para uma única temperatura nas mesmas condições de medição.

A UM é, em síntese, a variação da temperatura quando a distância do objeto de medição varia dentro do campo de visão da câmera, em condições de calibração.

\subsection{Resultado das incertezas intrínsecas}

A incerteza de medição devido a fontes internas foi obtida utilizando-se as fórmulas descritas neste artigo.

Utilizou-se para os cálculos da Tabela 1 as seguintes condições ambientais: temperatura do objeto $300{ }^{\circ} \mathrm{C}$, temperatura ambiente aproximadamente $20^{\circ} \mathrm{C}$ e umidade de $55 \%$.

Tabela 1: Incerteza devida somente a fontes internas da câmera térmica

\begin{tabular}{|c|c|c|c|c|}
\hline \multicolumn{5}{|c|}{ Temperatura do alvo a $300^{\circ} \mathrm{C}$} \\
\hline Grandeza & Valor $\left({ }^{\circ} \mathrm{C}\right)$ & $\begin{array}{c}\text { Avaliação da } \\
\text { incerteza }\end{array}$ & Distribuição & Incerteza Interna $\left({ }^{\circ} \mathrm{C}\right)$ \\
\hline EM & 6 & Tipo B & Retangular & 1,7 \\
\hline EGR & 0,0093 & Tipo B & Retangular & 0,0093 \\
\hline RTD & 0,0084 & Tipo B & Retangular & 0,0048 \\
\hline ET & 3,75 & Tipo B & Retangular & 1.08 \\
\hline $\mathrm{RE}$ & 0,03 & Tipo A & Normal & 0,0055 \\
\hline UM & 0,3 & Tipo B & Retangular & 0,17 \\
\hline
\end{tabular}


A Figura 3 descreve a relação entre a temperatura do objeto e sua respectiva incerteza interna, considerando condições ideais de medição.

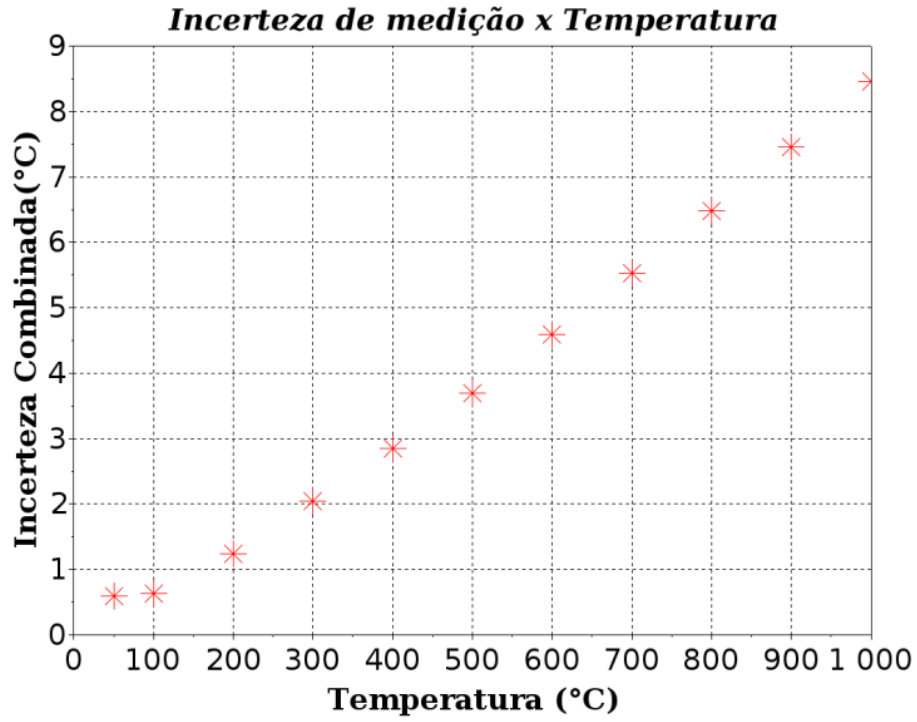

Figura 3: Relação entre a incerteza de medição interna e a temperatura do objeto.

A Figura 4 representa a relação entre a temperatura e o EGR.

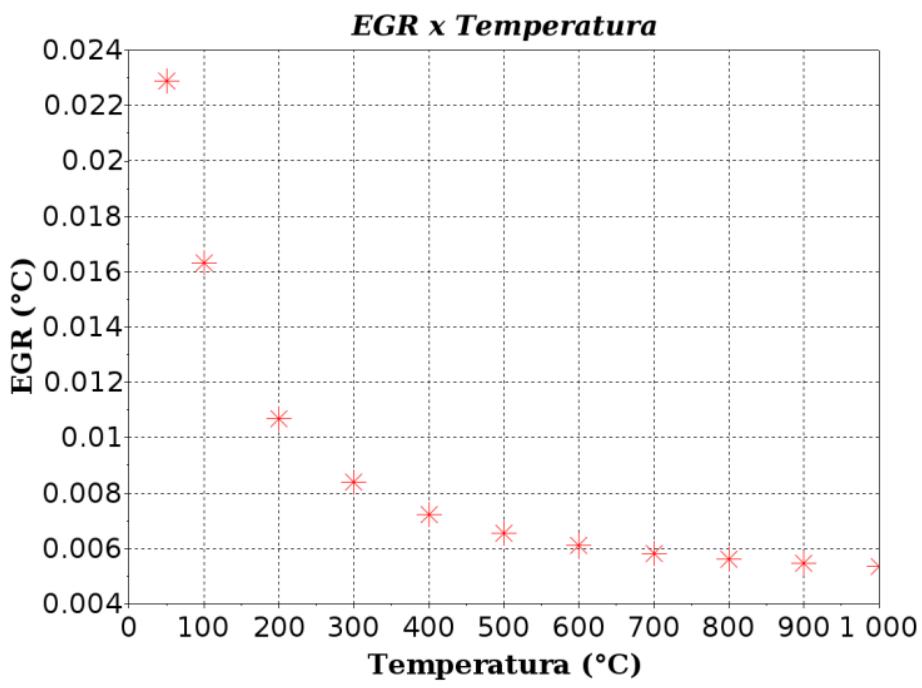

Figura 4: Relação entre o NGE e a temperatura do objeto. 


\section{CONCLUSÕES}

Observando-se a Figura 3 e a Tabela 1 pode-se constatar que o erro mínimo se sobressai diante das outras componentes de incerteza interna, isso porque o seu valor é superior tornando as outras contribuições insignificantes para a incerteza de medição.

Logo, pode-se concluir que o EM pode ser utilizado por usuários como uma estimativa da incerteza de medição se as medições forem realizadas em condições de calibração, o que permite que os usuários utilizem as câmeras térmicas com uma boa exatidão e possam fazer comparações, utilizando a incerteza de diferentes câmeras.

É perceptível também, ao se observar a Figura 4, que o valor do EGR diminui à medida que a temperatura do objeto aumenta, o que permite concluir que o erro gerado pelo ruído se torna menos significativo ao passo que a temperatura vai se tornando mais elevada.

Os resultados obtidos nesse artigo representam um esforço inicial desenvolvido pelo Inmetro no sentido de implementar futuras calibrações e comparações de termovisores utilizados por inúmeras indústrias, em especial, as de petróleo e gás, que utilizam esta tecnologia para a medição de temperatura.

As perspectivas futuras para o trabalho são: o desenvolvimento da incerteza de medição "completa", ou seja, que contemple as fontes de incertezas ditas como externas (emissividade, transmitância, temperatura ambiente e outras que não foram citadas no artigo), estudos acerca da confiabilidade do parâmetro accuracy quando utilizado em condições de medição desfavoráveis e a elaboração de um método de calibração que garanta a confiabilidade metrológica que as indústrias de petróleo e gás necessitam.

\section{AGRADECIMENTOS}

Agradecemos ao Inmetro pela utilização de sua infraestrutura de pesquisa e pela qualidade do ensino ofertado pelo Curso Técnico em Metrologia e ao PFRH (Programa de Formação de Recursos Humanos, da Petrobras) pela liberação de bolsa de estudo.

\section{REFERÊNCIAS}

Bureau International des Poids et Mesure (2012). International vocabulary of metrology: Basic and general concepts and associated terms (VIM).

Chrzanowski, K., Fischer, J., \& Matyszkiel, R. (2000). Testing and evaluation of thermal cameras for absolute temperature measurement. Optical Engineering, 39(9), 2535-2544.

Chrzanowski, K., Matyszkiel, R., Fischer, J., \& Bareła, J. (2001). Uncertainty of temperature measurement with thermal cameras. Optical Engineering, 40(6), 1106-1114.

Clausing, L. Pro tips: For using thermal imagers to examine tanks and vessels. Disponível em http://www.seacoastinspections.com/tanksandvessels.pdf 
Flir. Application story: Thermal imaging cameras for flare monitoring. Disponível em http://www.flir.com/uploadedFiles/CS_EMEA/Application_Stories/Media/Downloads/Flare_EN.p df?_ga=1.204873880.90497952.1465925220

Fluke (2000). Applications for thermal Imagers: Thermal process monitoring. Disponível em http://support.fluke.com/find-sales/download/asset/2526394_a_6251_eng_w.pdf

Gaussorgues, G. (1994). Infrared Thermography. doi:10.1007/978-94-011-0711-2

McEvoy, H., Simpson, R., \& Machin, G. Review of current thermal imaging temperature calibration and evaluation facilities, practices and procedures, across EURAMET (European Association of National Metrology Institutes). Proc. of QIRT, 9.

Neto, A. (2004). Noções sobre termografia curso: inspeções de equipamentos e instalações Petrobras. Rio de Janeiro: Íntegra.

Instituto Nacional de Metrologia, Qualidade e Tecnologia (2012). Vocabulário internacional de termos fundamentais e gerais de metrologia. Rio de Janeiro.

Xavier, J. (2005). Manutenção preditiva caminho para a excelência. Minas Gerais: Tecém. 